\title{
Fluorescent RNA tags: current and future applications
}

\author{
Sven Hennig*,1 (iD) \& Saskia Neubacher*,1 \\ ${ }^{1}$ Department of Chemistry \& Pharmaceutical Sciences, Vrije Universiteit Amsterdam, De Boelelaan 1108, Amsterdam 1081 HZ, The \\ Netherlands \\ *Authors for correspondence: s.a.neubacher@vu.nl
“The past 8 years of research on FLAPs have broadened the panel for in vitro and in vivo applications and facilitated a general implementation of the technique"

First draft submitted: 12 July 2019; Accepted for publication: 2 August 2019; Published online: 16 October 2019

Keywords: FLAP • fluorescent light-up aptamer $\bullet$ RNA assay $\bullet$ RNA imaging $\bullet$ RNA labeling $\bullet$ RNA-protein interaction - RNA-RNA interaction

The rising interest in the field of RNA research, especially regarding diverse (short and long) noncoding RNAs, demands for novel techniques to identify, functionally characterize and also chemically target these molecules [1,2]. Fluorescent light-up aptamers (FLAPs) are RNA sequences that bind to small molecules and thereby enhance their intrinsic fluorescence significantly [3]. They can be encoded on the DNA level and are then either transcribed in the cell and functionalized as FLAPs on the RNA level, or they can be in vitro transcribed and purified for further applications. FLAPs have been known since the discovery of a fluorescence-enhancing RNA aptamer for malachite green in 2003 [4]. However, it was only in 2011 that they gained importance when Paige and colleagues published an RNA aptamer that binds the cell-permeable and nontoxic derivative (Z)-4-(3,5-difluoro-4-hydroxybenzylidene)1,2-dimethyl-1H-imidazol-5(4H)-one (DFHBI) of the chromophore 4-hydroxybenzlidene imidazolinone (HBI) found in GFP [5]. This combination of RNA and small molecule, called Spinach, caused a plethora of follow-up publications focusing on either the variation or the optimization of different physicochemical features, resulting in RNA-fluorogen pairs named Baby Spinach, Broccoli, Corn or Mango, and others [6]. Currently, there are various small-molecule chromophores each in combination with a specific RNA aptamer sequence available. The most important variations appear within their spectral characteristics, the chemical nature of the chromophore, the size and stability of the formed RNA aptamer and their stoichiometry [3].

The past 8 years of research on FLAPs have broadened the panel for in vitro and in vivo applications and facilitated a general implementation of the technique. In the specific case of Mango, where the fluorogen carries a biotin moiety, the tag can be used to affinity pull down and purify fused RNA of interest, but also associated proteins (or RNAs), facilitating the identification of novel endogenous RNA-protein complexes. Tagging RNAs of interest to FLAPs has been used in early in vivo applications for highly abundant RNAs, such as the 5S ribosomal RNA (rRNA) or U6 snRNA [5]. Detection and localization of the tagged RNA of interest and quantitative measurements of polymerase III transcription in live mammalian cells were shown [7]. More recently, improved FLAPs were successfully used to detect low-abundance RNAs in vitro (FASTmiR), thereby substituting labor intensive methods (e.g. northern blotting, DNA-nanosilver clusters or in situ hybridization) and also in live cell imaging [8,9]. This allowed for quantitative and dynamic studies of mRNA transcription in cells. To measure mRNA and protein levels simultaneously, a FLAP can also be fused to a protein-encoding mRNA. A study where red fluorescent protein (mRFP1) was analyzed in Escherichia coli indicates that RNAs have a much higher turnover in cells than proteins [10]. FLAPs can also be fused to other aptamers that can then selectively bind to a target molecule (such as EGFR) to label cell surfaces [11].

Attached to so-called sensor RNAs, FLAPs enable the detection of biomolecules, such as metabolites, proteins or RNAs. Upon binding of the analyte to the sensor unit, a structural rearrangement causes the FLAP unit to fold and bind its fluorogen, which then results in fluorescence increase. These bifunctional RNAs have been used to 
detect many metabolites in vitro or in vivo, including adenosine triphosphate (ATP), adenosine $5^{\prime}$-diphosphate (ADP), flavin mononucleotide (FMN), cyclic-di-nucleotide monophosphates (e.g. c-di-GMP, c-di-AMP, c-AMPGMP), $S$-adenosyl-l-homocysteine (SAH) and $S$-adenosylmethionine (SAM), theophylline (TH) and thiamine 5 '-pyrophosphate (TPP) [12]. Similar approaches were used to detect and image proteins (such as streptavidin and MS2 coat protein) [13] and RNAs (mRNAs and micro RNAs) [8]. FLAPs are also used as tools in high-throughput screening, whereas their utilization is currently rather limited. In one example, the activity of an RNA-modifying enzyme was screened using a chemically modified fluorogen that simply causes the FLAP to light-up upon enzyme activity. For sure, great challenges lie ahead of high-throughput screening utilization of FLAPs, but the first steps have been made and clearly a great potential is hidden in this underexplored area [14].

Interactions between biomacromolecules are of great importance both for the understanding of cellular functions and also for the screening of potential interaction modulators [15,16]. A split version of the aptamer dBroccoli was shown to be able to reassemble and generate fluorescence [17]. Using this system cellular RNA-RNA interactions could be detected directly, and further downstream using a reporter gene as readout. In the Corn aptamer, homodimerization of the aptamer is a prerequisite for fluorogen binding and hence fluorescence [18]. This particular feature could potentially be used to build a system for the detection of RNA-RNA interactions. However, it is still unclear whether the homodimerization could prove to be too strong for this application in its current state. While most applications use fluorescence intensity as readout, in the context of intermolecular interactions, proximity-based Förster resonance energy transfer (FRET) is also a possible readout that can offer advantages. In the so-called 'apta-FRET', Spinach and Mango were fused together and used for small-molecule sensing and target RNA sensing [19]. This demonstrates the potential of FLAPs, especially to study bimolecular interactions in vivo. In principle, FRET can also occur between FLAPs and other fluorophores. In combination with protein fluorophores, the detection of RNA-protein interactions becomes possible. Spinach as a green fluorophore was used together with mCherry as a red protein fluorophore in a FRET-based system to detect and quantify the interaction between pp7-RNA and the PP7-coat-protein [20]. This is the first example that shows in principle a genetically encodable protein-RNA detection system.

FLAPs require the external addition of fluorogens, which is one of the major restrictions of their applications. In vivo, FLAPs are therefore currently limited to cultured cells. However, with growing knowledge in the field of RNA and its tertiary structures, it might be possible to generate intrinsic fluorescence directly from RNA. Until then, further research will be needed to improve properties of FLAPs, such as stability and fluorescence brightness. Being a genetically encodable fluorescent tag, FLAPs already offer many areas of research a tool to monitor RNA transcription, processing and trafficking. The first examples now have shown that this tool can be expanded to investigate interactions between RNA and RNA or RNA and proteins for basic and applied research.

\section{Financial \& competing interests disclosure}

The authors have no relevant affiliations or financial involvement with any organization or entity with a financial interest in or financial conflict with the subject matter or materials discussed in the manuscript. This includes employment, consultancies, honoraria, stock ownership or options, expert testimony, grants or patents received or pending, or royalties.

No writing assistance was utilized in the production of this manuscript.

\section{References}

1. Birney E, Stamatoyannopoulos JA, Dutta A et al. Identification and analysis of functional elements in $1 \%$ of the human genome by the ENCODE pilot project. Nature 447(7146), 799-816 (2007).

2. Anastasiadou E, Jacob LS, Slack FJ. Non-coding RNA networks in cancer. Nat. Rev. Cancer 18(1), 5-18 (2017).

3. Neubacher S, Hennig S. RNA structure and cellular applications of fluorescent light-up aptamers. Angew. Chem. Int. Ed. Engl. 57, 2-17 (2018).

4. Babendure JR, Adams SR, Tsien RY. Aptamers switch on fluorescence of triphenylmethane dyes. J. Am. Chem. Soc. 125(48), 14716-14717 (2003).

5. Paige JS, Wu KY, Jaffrey SR. RNA mimics of green fluorescent protein. Science 333, 642-646 (2011).

6. Truong L, Ferré-D’Amaré AR. From fluorescent proteins to fluorogenic RNAs: tools for imaging cellular macromolecules. Protein Sci. 28(8), 1374-1386 (2019).

7. Strack RL, Disney MD, Jaffrey SR. A superfolding Spinach2 reveals the dynamic nature of trinucleotide repeat-containing RNA. Nat. Methods 10(12), 1219-1224 (2013). 
8. Huang K, Doyle F, Wurz ZE et al. FASTmiR: an RNA-based sensor for in vitro quantification and live-cell localization of small RNAs. Nucleic Acids Res. 45(14), 1-13 (2017).

9. Shin I, Ray J, Gupta V et al. Live-cell imaging of Pol II promoter activity to monitor gene expression with RNA IMAGEtag reporters. Nucleic Acids Res. 42(11), 1-9 (2014).

10. Pothoulakis G, Ceroni F, Reeve B, Ellis T. The Spinach RNA aptamer as a characterization tool for synthetic biology. ACS Synth. Biol. 3(3), 182-187 (2014).

11. Tan X, Constantin TP, Sloane KL, Waggoner AS, Bruchez MP, Armitage BA. Fluoromodules consisting of a promiscuous RNA aptamer and red or blue fluorogenic cyanine dyes: selection, characterization and bioimaging. J. Am. Chem. Soc. 139, 9001-9009 (2017).

12. Sun Z, Nguyen T, McAuliffe K, You M. Intracellular imaging with genetically encoded RNA-based molecular sensors. Nanomaterials 9(2), 233 (2019).

13. Song W, Strack RL, Jaffrey SR. Imaging bacterial protein expression using genetically encoded RNA sensors. Nat. Methods 10(9), 873-875 (2013).

14. Svensen N, Jaffrey SR. Fluorescent RNA aptamers as a tool to study RNA-modifying enzymes. Cell Chem. Biol. 23(3), 415-425 (2016).

15. Khalil AM, Rinn JL. RNA-protein interactions in human health and disease. Semin. Cell Dev. Biol. 22(4), 359-365 (2011).

16. Guan L, Disney MD. Recent advances in developing small molecules targeting RNA. ACS Chem. Biol. 7(1), $73-86$ (2012).

17. Alam KK, Tawiah KD, Lichte MF, Porciani D, Burke DH. A fluorescent split aptamer for visualizing RNA - RNA assembly in vivo. ACS Synth. Biol. 6, 1710-1721 (2017).

18. Jepsen MDE, Sparvath SM, Nielsen TB et al. Development of a genetically encodable FRET system using fluorescent RNA aptamers. Nat. Commun. 9(1), 1-10 (2018).

19. Warner KD, Sjekloa L, Song W, Filonov GS, Jaffrey SR, Ferré-D’Amaré AR. A homodimer interface without base pairs in an RNA mimic of red fluorescent protein. Nat. Chem. Biol. 13(11), 1195-1201 (2017).

20. Roszyk L, Kollenda S, Hennig S. Using a specific RNA-protein interaction to quench the fluorescent RNA spinach. ACS Chem. Biol. 12(12), 2958-2964 (2017). 